\title{
Differences in short-term training for interaural phase difference discrimination between two different forced-choice paradigms
}

Andrew King ${ }^{1)}$, Kathryn Hopkins and Christopher J. Plack

School of Psychological Sciences, The University of Manchester, Manchester, M13 9PL, United Kingdom

(Received 14 January 2013; revised 2 August 2013; accepted 9 August 2013)

Improvement in interaural phase difference (IPD) discrimination over two to three hours was compared for two two-alternative forced-choice paradigms: a three-interval paradigm, in which the IPD was in interval two or three, and a paradigm with two intervals of four stimuli in which the IPD was in the second and fourth stimuli of one interval (AAAA vs. ABAB). The difference in performance between the beginning and end of the testing period was smaller for the two-interval paradigm, supporting the use of this paradigm for fast measurement of discrimination thresholds without the need for a long period of training.

PACS numbers: 43.66.Yw, 43.66.Pn, 43.66.Mk [TD]

Pages: 2635-2638

\footnotetext{
${ }^{1)}$ Author to whom correspondence should be addressed. Email: andrew.king-5@ postgrad.manchester.ac.uk
} 


\section{INTRODUCTION}

Performance on psychophysical discrimination tasks often improves with increasing number of trials (Wright and Fitzgerald, 2001; Hawkey et al., 2004; Amitay et al., 2006), due to learning attributes of the stimuli (perceptual learning) and/or procedural learning. Learning may reduce the accuracy of estimates from repeated measurements, and different learning rates for different conditions, listeners, or tasks can introduce bias. Training participants until performance reaches asymptote can minimize these problems, but this can take thousands of trials (Hafter and Carrier, 1970). Long training periods are not an option if the available time to test each listener is short, for example, in clinical assessment. Hence, it is important to find a procedure that minimizes the change in performance over time.

Hopkins and Moore (2010) and Moore and Sęk (2009) described a two-interval discrimination paradigm $(8 \mathrm{~S} 2 \mathrm{~A})$ for which they reported minimal training effects. One interval (chosen at random) contained four non-target stimuli (AAAA). The other interval contained alternating non-target and target stimuli (ABAB). Hopkins and Moore (2010) found no significant improvement in pure-tone, interaural phase difference (IPD) discrimination over the 16 adaptive tracks for each of the three frequencies that were tested. Moore and Sęk (2009) found no significant difference in discrimination of harmonic and frequency-shifted bandpass filtered complex tones over two, two-hour training sessions for 10 trained and 10 untrained listeners. However, neither study compared the 8S2A paradigm with any other presentation paradigm, so it is not clear whether the paradigm, or some other aspect of the experimental procedure, produces stable performance.

Here the effect of training on IPD discrimination performance for the 8S2A paradigm was compared to that for the three-interval, two-alternative (3I2A) paradigm described by Lacher-Fougère and Demany (2005).

\section{METHOD}

\section{A. Stimuli}

Three types of stimuli were used: pure tones (PT IPD), amplitude modulated (AM) tones with the IPD in the temporal fine structure (TFS IPD), and AM tones with an IPD in the temporal envelope (Envelope IPD). The AM tones were specified by equation 1 :

$$
s(t)=\sin \left(2 \pi F_{c a r} t+\varphi_{c a r}\right) \cdot\left[1+\sin \left(2 \pi F_{\text {mod }} t+\varphi_{\text {mod }}\right)\right] \text {, }
$$

where $F_{c a r}$ is the carrier frequency, $F_{m o d}$ is the modulation frequency, $\varphi_{c a r}$ is the carrier phase, $\varphi_{\text {mod }}$ is the modulation phase and $t$ is the sample index. Differences in $\varphi_{c a r}$ and $\varphi_{\text {mod }}$ between ears (referred to here as $\delta$ ) produced IPDs in the TFS (TFS IPD) and envelope (Envelope IPD), respectively. IPDs were created by introducing a positive starting phase in one ear and zero starting phase in the other. The frequency of the pure tones and $F_{c a r}$ for the AM tones was $500 \mathrm{~Hz}$. For the AM tones $F_{\text {mod }}$ was $20 \mathrm{~Hz}$. Stimuli were presented at a level of $80 \mathrm{~dB}$ SPL. The stimulus duration was $500 \mathrm{~ms}$, including 50-ms raised-cosine onset and offset ramps, which were synchronous across ears. The inter-stimulus silent interval was $500 \mathrm{~ms}$ for both paradigms. For the 8S2A paradigm the four stimuli in each interval were separated by $50 \mathrm{~ms}$ of silence. Stimuli were created in MATLAB (Mathworks) at a sample rate of $48 \mathrm{kHz}$ 
and output via a Creative E-MU 0202 USB 24-bit soundcard and Sennheiser HD 650 circumaural headphones within a double-walled listening booth.

\section{B. Procedure}

For the 3I2A paradigm, listeners indicated whether the second or third interval differed from the first interval (a diotic reference stimulus) by pressing ' 2 ' or ' 3 ' on a computer keyboard. One of the last two intervals (chosen at random) had an IPD of $\delta$; the other was identical to the diotic reference. For the 8S2A paradigm, each interval contained four stimuli. In one interval (chosen at random) the stimuli were all diotic (AAAA). In the other interval, the first and third stimuli in the sequence were also diotic, but the second and fourth contained an IPD of $\delta$ (ABAB). Listeners indicated whether the ABAB interval was first or second by pressing ' 1 ' or ' 2 ' on the keyboard. An onscreen light indicated each interval and other lights provided feedback. At the beginning of each run, $\delta$ was set to $180^{\circ}$. A two-down, one-up adaptive method was used to track $70.7 \%$ correct on the psychometric function. The initial step size was a factor of $1.25^{2}$. After four reversals, the step size was reduced to 1.25 for a further 10 reversals. The geometric mean of the values of $\delta$ at the last 10 reversals was taken as the threshold $\delta$.

Thirty-six normal-hearing listeners (18-35 years, mean=23) were tested (audiometric thresholds $<=20 \mathrm{~dB}$ HL between 0.25 and $8 \mathrm{kHz}$, and $<10 \mathrm{~dB}$ difference in thresholds between ears at $500 \mathrm{~Hz}$ ). Listeners were randomly allocated into six groups of six, with each group allocated a different combination of paradigm and stimulus. Twenty-two runs were completed over a total period of approximately two hours with the 3I2A paradigm and three hours with the 8S2A paradigm. The majority of listeners tested with the 8S2A paradigm completed the experiment over two sessions whilst the listeners tested with the 3I2A paradigm were more likely to complete the experiment in one session.

\section{RESULTS}

Figure 1 shows the geometric mean IPD discrimination thresholds for each group as a function of run number. For analysis, the geometric mean of the first four values (pre training) was compared with the geometric mean of the last four values (post training) for each listener. The listener-group geometric means for these measures are shown in Fig. 2. All analyses were performed on the log-transformed data values to satisfy the assumption of normality. A mixed model ANOVA was performed with training (pre and post) as the within-subjects factor, and paradigm (3I2A and 8S2A) and training stimulus (envelope IPD, TFS-IPD and PT-IPD) as between-subjects factors. Thresholds were lower following training $[F(1,30)=10.0, p=0.004]$, and listeners tested using the $8 \mathrm{~S} 2 \mathrm{~A}$ paradigm had lower mean thresholds than listeners tested using the $3 \mathrm{I} 2 \mathrm{~A}$ paradigm $[F(1,30)=5.3, p=0.03]$. There was no significant effect of stimulus. 


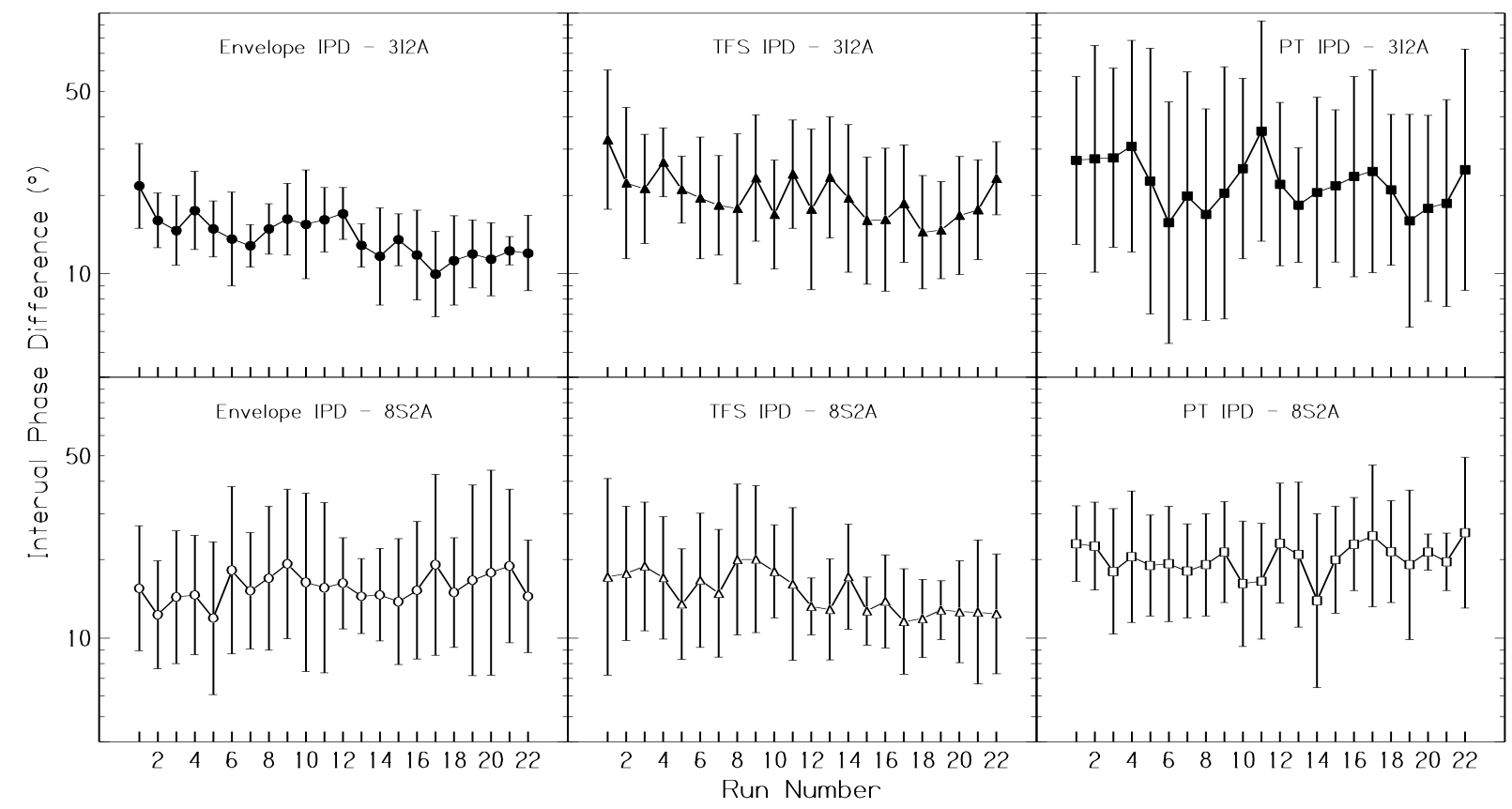

FIG.1: The geometric mean IPD discrimination thresholds for each group of six listeners as a function of run number. Error bars show $95 \%$ confidence intervals.

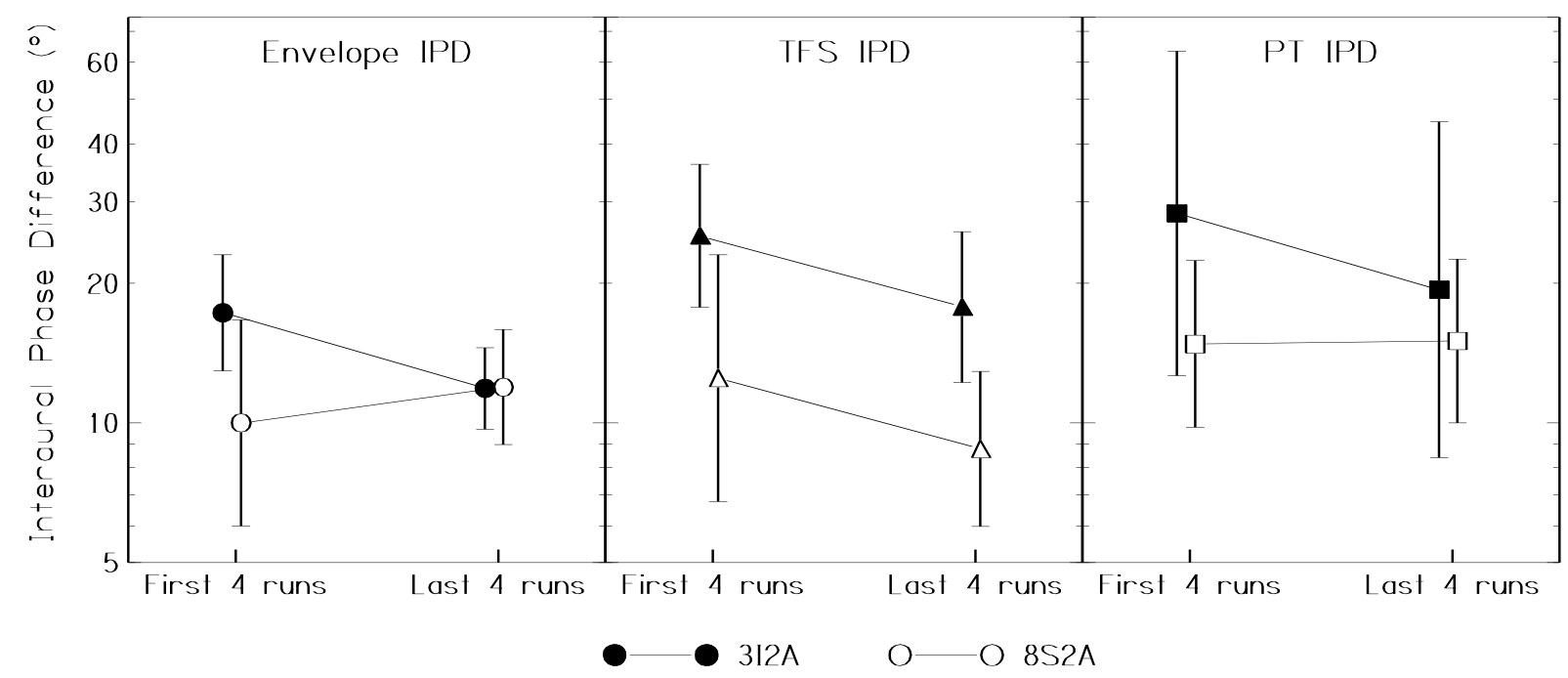

FIG. 2: The geometric mean IPD discrimination thresholds obtained from the first four adaptive runs and the last four adaptive runs completed in each training condition, averaged across listeners. Data are offset on the abscissa for visual clarity. Error bars show $95 \%$ confidence intervals.

The interaction between training and paradigm was significant $[F(1,30)=5.6, p=0.02]$. To examine the effect of training for the two paradigms separately, thresholds were collapsed across stimuli and paired t-tests were performed for each paradigm. Thresholds for the two paradigms are shown in the left panel of Fig. 3 by symbols joined by solid lines. Listeners in the $8 \mathrm{~S} 2 \mathrm{~A}$ paradigm groups improved only slightly ( $\mathrm{pre}=12.3^{\circ}, \mathrm{SD}=1.9^{\circ}$; post $=11.6^{\circ}$, $\mathrm{SD}=1.9^{\circ}$ ) and the improvement was not significant. Listeners in the $3 \mathrm{I} 2 \mathrm{~A}$ paradigm groups showed a greater improvement ( $\mathrm{pre}=23.2^{\circ}, \mathrm{SD}=2.0^{\circ}$; post $=16.0^{\circ}, \mathrm{SD}=2.0^{\circ}$ ), which was significant $[t(34)=3.7, p=0.002]$. None of the other interactions in the ANOVA were significant $(p>0.05)$. 


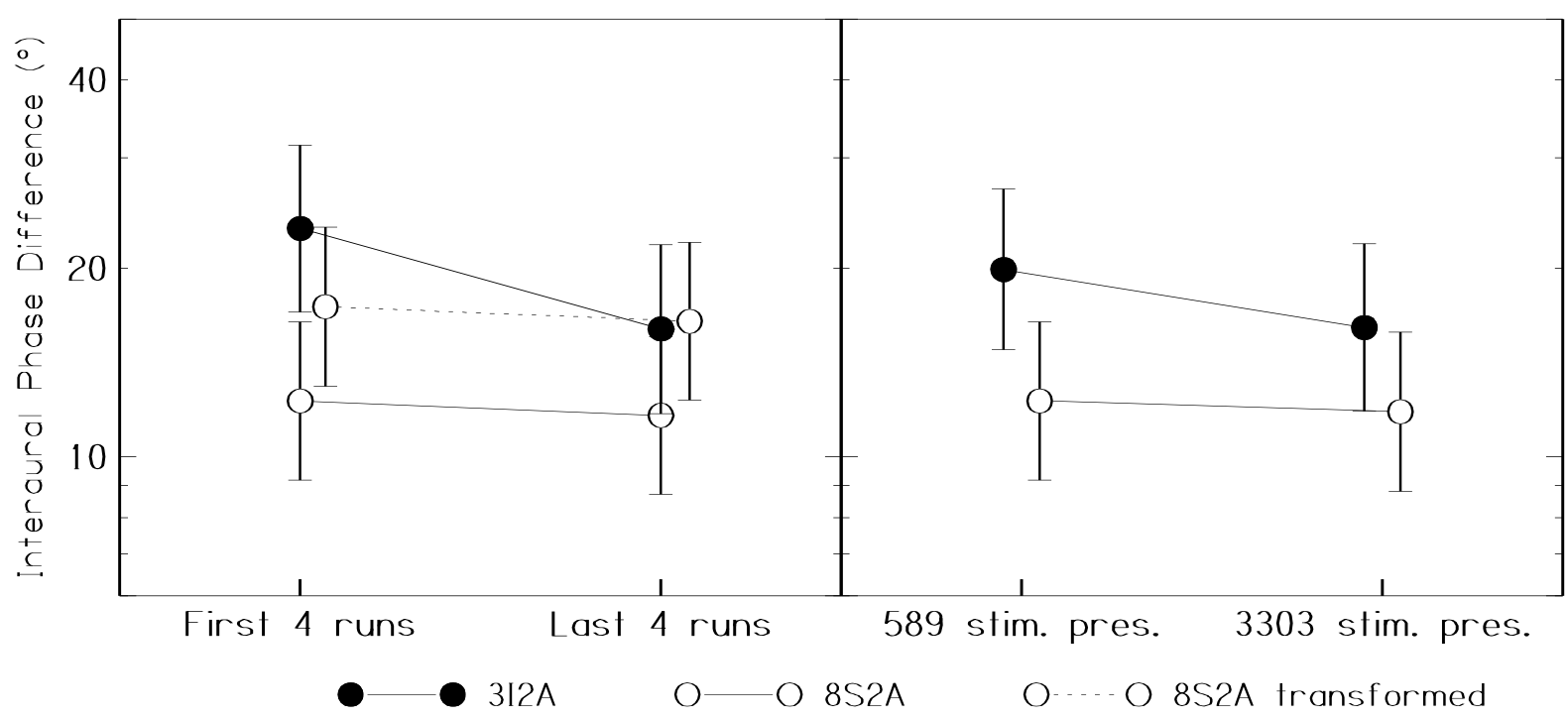

FIG. 3: In the left panel the symbols joined by solid lines show the geometric means of the first four runs and the last four runs in the two paradigm conditions, averaged across stimuli and listeners. The symbols joined by the dashed line show transformed $8 \mathrm{~S} 2 \mathrm{~A}$ data. The right panel shows the geometric means of the four runs up to and including the run that exceeded 589 and 3303 stimulus presentations, averaged across stimuli and listeners. Data are offset on the abscissa for clarity. Error bars show 95\% confidence intervals.

In the first ANOVA, thresholds for the $8 \mathrm{~S} 2 \mathrm{~A}$ paradigm were significantly lower than for the 3I2A paradigm. Both paradigms were two alternative forced-choice tasks, but the 8S2A paradigm had twice as many observations as the $3 \mathrm{I} 2 \mathrm{~A}$ paradigm that contributed to the discrimination decision (i.e. with a priori probabilities of being either reference or target). dprime ( $\mathrm{d}^{\prime}$ ) increases by the square root of the number of times a trial is repeated before a forced-choice decision is made (Swets et al., 1959), and this might explain why lower thresholds were observed for the 8S2A paradigm. Assuming independent internal noise and equal variance in the distributions of reference and target stimuli (Green and Swets, 1974), d' would be expected to be $\sqrt{2}$ times greater for the 8 S2A paradigm than for the 3 I2A paradigm. $d^{\prime}$ increases linearly with IPD (Hafter and Carrier, 1972). Hence, to compensate for this effect, the 8S2A IPD thresholds (calculated over four runs) were multiplied by $\sqrt{ } 2$ before being log-transformed. The dashed line in Fig. 3 shows the mean transformed $\delta$. Notice that pre-training performance for the $8 \mathrm{~S} 2 \mathrm{~A}$ paradigm was similar to post-training performance for the $3 \mathrm{I} 2 \mathrm{~A}$ paradigm. The mixed model ANOVA was repeated using these transformed 8S2A thresholds. The main effect of paradigm was not significant. The main effects of training and stimulus, and the interaction effects were unchanged.

The 3I2A paradigm had only three stimuli per trial and the 8S2A paradigm had eight. To take account of this difference, non-transformed mean thresholds were compared after approximately equal numbers of stimulus presentations. Pre training was defined as the runs up to and including the run in which listeners had heard 589 stimulus presentations (the mean number presented in the first four runs of the 8S2A paradigm) and post training was defined as the four runs up to and including 3303 stimulus presentations (the smallest total number of stimuli heard by all listeners). The right panel of Fig. 3 shows these data for the two paradigms. The first mixed-model ANOVA was repeated using these threshold estimates. 
The main effect of training was significant $[\mathrm{F}(1,30)=11.06, \mathrm{p}=0.002]$. However, there were no significant main effects of paradigm or of stimulus. The interaction between training and paradigm was significant $[\mathrm{F}(1,30)=5.09, \mathrm{p}=0.032]$. All other interactions were nonsignificant.

The difference between thresholds at the end of session one and at the start of session two (Mean $=-1.0^{\circ}, \mathrm{SD}=1.9^{\circ}$ ), for those who completed the study in two sessions, was not significantly greater than the difference between the $11^{\text {th }}$ and $12^{\text {th }}$ runs (Mean $=-0.8^{\circ}$, $\left.\mathrm{SD}=2.0^{\circ}\right)$ for those who completed the study in one session $(t=-0.76, p=0.45)$.

\section{DISCUSSION}

The PT-IPD thresholds collected using the 8S2A paradigm were $25 \%$ lower than the thresholds collected by Hopkins and Moore (2010) at $50 \mathrm{~dB}$ SL (the most similar condition to the PT-IPD condition tested here). The higher SLs in the current study may explain the lower IPD thresholds, but Hopkins and Moore (2010) report no evidence of a stimulus level effect between 30 and $50 \mathrm{~dB}$ SL. The Envelope- and TFS-IPD thresholds collected using the 3I2A paradigm were approximately $50 \%$ and $470 \%$ higher, respectively, than the thresholds reported by Lacher-Fougère and Demany (2005) using similar stimuli. The current study and Hopkins and Moore (2010) used inexperienced listeners, but Lacher-Fougère and Demany (2005) do not report listener experience. Therefore, previous experience may have been a contributing factor to the differences in IPD thresholds. Lacher-Fougère and Demany (2005) tested listeners over four, $1 \mathrm{~h}$ sessions, whereas the current study and Hopkins and Moore (2010) only used two sessions. This may affect training, but in the current study there were no significant differences in thresholds between listeners who completed the study in two sessions and those who completed it in one session.

The smaller effect of training for the 8S2A paradigm than the 3I2A paradigm supports the use of the $8 \mathrm{~S} 2 \mathrm{~A}$ paradigm for testing IPD discrimination thresholds (and potentially thresholds for other discrimination tasks) if stable performance is desired over a two- or three-hour time frame. Stable performance in the 8S2A paradigm may have occurred for two reasons. One explanation is that the task was very easy to learn, so either no training was needed to reach asymptote, or performance reached its asymptote within the first four runs. The alternative explanation is that insufficient training was provided for substantial improvement to occur during the course of the experiment. When adjusted for the greater total target duration, the $8 \mathrm{~S} 2 \mathrm{~A}$ thresholds pre training were similar to the $3 \mathrm{I} 2 \mathrm{~A}$ thresholds post training. This suggests that performance asymptotes quickly for the $8 \mathrm{~S} 2 \mathrm{~A}$ paradigm. The lack of training effect for the 8S2A paradigm is consistent with Moore and Sęk (2009) and Hopkins and Moore (2010).

In conclusion, IPD thresholds may not be comparable across the two paradigms without accounting for the different numbers of stimulus presentations per trial. However, the 8S2A paradigm appears better than the $3 \mathrm{I} 2 \mathrm{~A}$ paradigm for avoiding training effects and the potential variability and bias they may produce over the course of several hours of testing. Hence, the 8 S2A paradigm may be more appropriate for fast assessment of discrimination abilities.

\section{ACKNOWLEDGMENTS}


This work was supported by Oticon A/S and the Medical Research Council (UK) (ref G1001609).

Amitay, S., Irwin, A., Hawkey, D. J., Cowan, J. A., and Moore, D. R. (2006). "A comparison of adaptive procedures for rapid and reliable threshold assessment and training in naive listeners," J. Acoust. Soc. Am. 119, 1616-1625.

Green, D. M., and Swets, J. A. (1974). Signal Detection Theory and Psychophysics (Krieger, New York), pp. 53-85, 154-161.

Hafter, E. R., and Carrier, S. C. (1970). "Masking-level differences obtained with a pulsed tonal masker," J. Acoust. Soc. Am. 47, 1041-1047.

Hafter, E. R., and Carrier, S. C. (1972). "Binaural interaction in low-frequency stimuli: the inability to trade time and intensity completely," J. Acoust. Soc. Am. 51, 1852-1862.

Hawkey, D. J., Amitay, S., and Moore, D. R. (2004). "Early and rapid perceptual learning," Nat. Neurosci. 7, 1055-1056.

Hopkins, K., and Moore, B. C. J. (2010). "Development of a fast method of measuring sensitivity to temporal fine structure information at low frequencies.," Int. J. Audiol. 49, 940-946.

Lacher-Fougère, S., and Demany, L. (2005). "Consequences of cochlear damage for the detection of interaural phase differences," J. Acoust. Soc. Am. 118, 2519-2526.

Moore, B. C. J., and Sek, A. (2009). "Development of a fast method for determining sensitivity to temporal fine structure," Int. J. Audiol. 48, 161-171.

Swets, J. A., Shipley, E. F., McKey, M. J., and Green, D. M. (1959). "Multiple observations of signals in noise," J. Acoust. Soc. Am. 31, 514-521.

Wright, B. A., and Fitzgerald, M. B. (2001). "Different patterns of human discrimination learning for two interaural cues to sound-source location," Proc. Natl. Acad. Sci. U. S. A. 98, 12307-12312. 